\title{
Erratum to: Integrated gene networks in breast cancer development
}

\author{
Ivana Ratkaj • Emil Stajduhar • Srdan Vucinic • \\ Sime Spaventi • Hrvojka Bosnjak • Kresimir Pavelic • \\ Sandra Kraljevic Pavelic
}

Published online: 12 October 2010

(C) Springer-Verlag 2010

\section{Erratum to: Funct Integr Genomics (2010) 10:11-19 DOI 10.1007/s10142-010-0159-2}

The original version of this article, unfortunately, contained several mistakes.

In page 14 of the article, there was a mistake in the last paragraph explaining the result.

The sentence was:

(...)These results are in concordance with previously published data and support the hypothesis about the importance of hGH in breast cancer progression, although its production was significantly higher in ductal invasive carcinoma in comparison with benign fibroadenoma
(...)

instead of

(...) These results are in concordance with previously published data and support the hypothesis about the importance of hGH in breast cancer progression, although its production was significantly higher in benign fibroadenoma in comparison with ductal invasive carcinoma(...)

The Electronic Supplementary Material was also was not published in its complete form. The updated version is shown in the Electronic Supplementary Material provided.

The online version of the original article can be found at http://dx.doi. org/10.1007/s10142-010-0159-2.

Electronic supplementary material The online version of this article (doi:10.1007/s10142-010-0194-z) contains supplementary material, which is available to authorized users.

I. Ratkaj $(\bowtie) \cdot$ K. Pavelic $\cdot$ S. Kraljevic Pavelic Department of Biotechnology, University of Rijeka,

Trg brace Mazuranica 10,

51000 Rijeka, Croatia

e-mail: iratkaj@irb.hr

E. Stajduhar

Department of Radiology, Clinical hospital "Sisters of Mercy",

Vinogradska cesta 29,

10000 Zagreb, Croatia

\section{S. Vucinic}

Laboratory for Systems Biomedicine,

Division of Molecular Medicine, Ruder Boskovic Institute,

Bijenicka cesta 54,

10000 Zagreb, Croatia
S. Spaventi

Croatian Academy of Sciences and Arts,

Zrinski trg 11,

10000 Zagreb, Croatia

H. Bosnjak

Department of Internal Medicine,

Clinical hospital "Sisters of Mercy",

Vinogradska cesta 29,

10000 Zagreb, Croatia 